International Tinnitus Journal. 2014;19(1):41-45.

\title{
The effect of circadian rhythm on the perceived tinnitus severity: A preliminary study
}

\author{
Thomas Zacharia ${ }^{1}$ \\ Judith James ${ }^{1}$ \\ Hari Prakash ${ }^{1}$ \\ Rukmangathan Thurvas Mohan ${ }^{1}$ \\ Bellur Rajashekhar ${ }^{1}$
}

\begin{abstract}
Introduction: Hearing sound that does not originate in the world outside the body is an experience every human has at one time or another in life. Circadian rhythm is common for all living organisms and nearly all physiological functions, especially sleep-wake cycles, exhibit circadian rhythmicity. A short survey conducted in the study center revealed that majority of the tinnitus subjects reported a difference in tinnitus severity across the day. So the current study focused on finding out the influence of circadian rhythm on tinnitus severity. Materials \& Methods: Study was conducted on 20 tinnitus subjects irrespective of age, gender, hearing status and type of tinnitus. Kannada version of MEQ was given to all the subjects to classify them to various circadian types. A visual analog scale was also given to rate the severity of the tinnitus in the morning and evening. Results: The result of the current study states that, there was a significant difference in the tinnitus severity for moderate morning type individuals in the morning and evening and not for the intermediate group. Conclusion: So we can conclude that, circadian rhythm has a strong association with the severity of the tinnitus.
\end{abstract}

Keywords: circadian rhythm, questionnaires, tinnitus.

\footnotetext{
${ }^{1}$ Department of Speech \& Hearing School of Allied Health Sciences (SOAHS) - Manipal University - Manipal - India. E-mail: thomasasIp@gmail.com/ judithjames91@gmail.com/h.prakash.p@gmail.com/tmrukmangathan@gmail.com/b.raja@manipal.edu

Institution: Department of Speech \& Hearing School of Allied Health Sciences (SOAHS).

Send correspondence to:

Thomas Zacharia

Department of Speech \& Hearing School of Allied Health Sciences (SOAHS), Manipal University, Manipal, Karnataka. Zip Code: 576104

Paper submitted to the ITJ-SGP (Publishing Management System) on January 24, 2015;

and accepted on March 31, 2015. cod. 188
} 


\section{INTRODUCTION}

Tinnitus is defined as a disorder of perceiving tones or noises in the absence of corresponding external acoustic stimul ${ }^{1}$. Hearing sound that does not originate in the world outside the body is an experience every human has at one time or another in life. The sounds heard ranges from popping, clicking, roaring, buzzing, and pure tones. It is one of the three most common and distressing otological problems, besides sensori-neural hearing loss and vertigo, causing various somatic and psychological disorders that interfere with the quality of life. Tinnitus is a subjective phenomenon that is difficult to evaluate objectively, with it being measured, quantified, and described only based on the responses of patients. Chronic tinnitus is a very common complaint in the adult population (> 50 years). Its impact on psychological well-being could be so severe that it has been identified as a risk factor for suicide in the elderly ${ }^{2}$.

According to the National Center for health statistics (1968), about $32 \%$ of all US adults report having tinnitus at one time or another. And about $6.4 \%$ of the same population characterizes tinnitus as severe or handicapping. It is also reported that tinnitus is more common in men than women and increase in prevalence with advancing age $^{3}$. Tinnitus affects around $10-15 \%$ of the total world population ${ }^{2}$. It was also observed that, $1-3 \%$ of the tinnitus sufferers experience the disorder to a degree that, their quality of life has been severely affected ${ }^{4}$. Younger males with tinnitus reported fewer health-related difficulties than middle-aged male patients and the older men. Younger and middle-aged women with tinnitus experienced more health-related problems than older women.

The distinction between the sound of tinnitus and the impact that, tinnitus may have on a person is important because they are essentially independent phenomena. According to Tyler \& Baker ${ }^{5}$, the difficulties faced by tinnitus sufferers includes difficulty getting to sleep, persistence of tinnitus, understanding speech, despair, frustration, depression, annoyance, irritation, inability to relax and poor concentration or confusion It was also found out that, tinnitus sufferers can have psychiatric disorders, anxiety disorders, depressive pathosis, sleep disturbances, misophonia, phonophobia, and hyperacusis ${ }^{6,7}$.

Circadian rhythm is common for all living organisms and nearly all physiological functions, especially sleep-wake cycles, exhibit circadian rhythmicity. Circadian rhythms are endogeneous and are seen in the absence of environmental time cues ${ }^{8}$. The suprachiasmatic nucleus (SCN), a structure located in the anterior hypothalamus is the site of master circadian clock $^{9}$. It consists of a network of approximately 20,000 neurons in human and exhibits a self-sustained circadian rhythm in neuronal firing ${ }^{10,11}$. The circadian rhythm controls various biological activities, such as sleep-wake cycle, body temperature, hormone secretion, and cell cycle regulation. The timing of these physiologic processes if altered, leads to changes in the phase relationship of rhythms to one another, which leads to internal desynchronization. Either disruption of the endogeneous circadian control mechanism or misalignment between internal circadian rhythms with the 24-hour outside environment would result in circadian rhythm disorders which has adverse consequences in sleep and many other processes including metabolism dysfunction, cognitive impairment, cardiovascular abnormalities, gastrointestinal and genitourinary dysfunctions ${ }^{12,13}$.

Morningness-Eveningness questionnaire ${ }^{14}$, circadian type inventory ${ }^{15}$, composite scale of morningness ${ }^{16}$, Lark-Owl chronotypes indicator ${ }^{17}$ and Munich chronotypes questionnaire ${ }^{18}$ are the most clinically used scales to classify the individuals into different circadian patterns. In the above mentioned scales, Morningness-Eveningness Questionnaire (MEQ) is the most commonly used scale to classify individuals into different circadian types. MEQ classifies individuals into 5 circadian types such as, definite morning, moderate morning, intermediate, moderate evening and definite evening.

A short survey conducted in the current study center revealed that around $65 \%$ of the tinnitus patients (with and without hearing loss) report a difference in tinnitus severity across the day. These subjects can be classified under two groups. First group in which, subjects perceive a greater severity of tinnitus in the morning and a reduced tinnitus severity in the evening. The second group manifest an opposite fashion were a reduced severity was seen in the morning and an increased severity in the evening. But the reason for this difference in tinnitus severity is given less importance from the clinical perspectives and is poorly studied and documented in the literature. So the current study focuses for the first time in literature, the effect of circadian rhythm on the perceived tinnitus severity in individuals of various chronotypes at two time points.

\section{METHOD}

The English version of morningness-eveningness questionnaire (MEQ) which was developed by Horne \& Ostberg (1975) was translated and standardized to Kannada prior to the commencement of the study (under review for publication). All the participants who complained of tinnitus irrespective of age, gender, hearing status, types of tinnitus were recruited for the study. Participants suffering from psychiatric illness, tinnitus secondary to emotional disturbances were excluded from the study. A basic audiological evaluation was 
carried out on all the participants including, pure tone audiometry (PTA), Immittance Audiometry and Oto Acoustic Emission (OAE).

The Kannada version of MEQ (MEQ-K) was administered on 30 native Kannada speaking tinnitus subjects with an age range of 18 to 70 years with a mean age of 48.5 years to identify their circadian type. Along with the MEQ-K, the participants were also given a Visual Analog Scale (VAS) of 10 being very less and 50 being severe to rate the severity of tinnitus in the morning and in the evening. An oral consent was taken from all the participants prior to the administration of the questionnaire and the participants were asked to voluntarily withdraw from the study any time without prior notification if they wish to do so.

The principle investigator analyzed all the 30 questionnaires to prune them and to classify the participants according to their chronotypes. All the statistical analysis was done using SPSS Statistics 17.0 (SPSS Inc. Chicago). Test of normality was carried out using Shapiro-Wilk to see whether the data follows normal distribution or not. A paired sample $t$ test was done to find out statistical significance between the severity of tinnitus in the morning and evening within the groups at $95 \%$ confidence interval (Cl).

\section{RESULTS}

After the analysis of all the 30 distributed questionnaires, 20 completely filled questionnaires were included for the further analysis. Based on the scores obtained from the MEQ-K, all 20 participants were classified into 2 chronotypes. Out of 20 participants, $8(40 \%)$ were categorized as intermediate types and $12(60 \%)$ were categorized as moderate morning type group (Figure 1). There were no participants under definite morning, definite evening and moderate evening. Audiological evaluation revealed that $65 \%$ of the participants had some sort of hearing loss with severity ranging from minimal to profound. Remaining 35\% of participants had normal hearing sensitivity based on the PTA, Immittance and OAE findings. Based on the results of Shapiro-Wilk test, it was understood that the obtained data followed a normal distribution. The intermediate group and moderate morning group has a normality score of $0.389 \& 0.002$ respectively in the morning. Both the groups have normality scores of $0.389 \& 0.004$ respectively in the evening.

Results of paired sample $t$ test revealed that there is a statistically significant difference in the severity of perceived tinnitus in the morning and evening by the moderate morning group individuals ( $p$ value $<0.01$ ). The mean severity of tinnitus was 25 with a standard deviation of 6.74 in the morning and in the evening the severity was 33 with a standard deviation of 6.51 for the moderate morning group participants (Figure 2). However, a statistically significant difference was not obtained for the intermediate group participants with respect to the tinnitus severity ( $p$ value $=1)$. The mean severity of tinnitus was 25 with a standard deviation of 15.16 in the morning and in the evening (Figure 2).

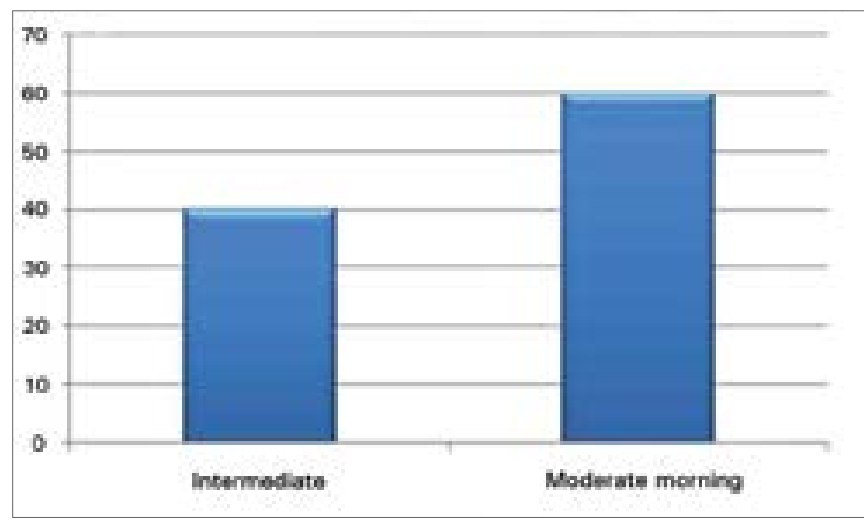

Figure 1. Percentage of participants under various circadian types.

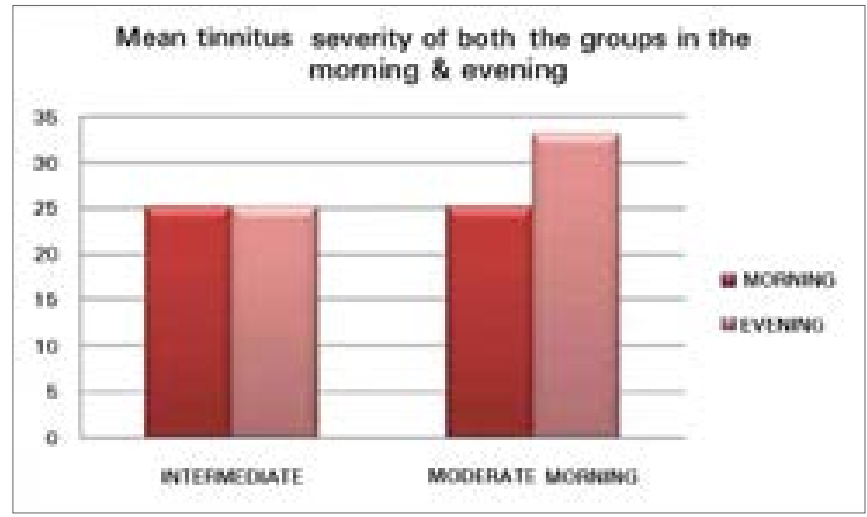

Figure 2. Mean tinnitus severity of both the groups in the morning and evening.

\section{DISCUSSION FIGURE 1. PERCENTAGE OF PARTICIPANTS UNDER VARIOUS CIRCADIAN TYPES.}

The aim of the current study is to find out the effect of circadian rhythm on the perceived tinnitus severity. For this, initially the authors adopted the most clinically used morningness-eveningness questionnaire (MEQ) to Kannada language. The statistical analysis revealed, there is a statistically significant difference in the severity of tinnitus perceived by the participants in the morning and evening time points. But this difference was only observed for moderate morning group individuals and not for the intermediate group. From the current study it was observed that, moderate morning type individuals had less severity of tinnitus in the morning and the tinnitus severity increased towards the evening. In the case of 
intermediate group, the severity of tinnitus remained the same throughout the day. Tinnitus loudness varies in individuals with respect to the extent of hearing loss and is different from one individual to another. But change in tinnitus loudness within an individual at two time points in a day was not given much interest in the past. According to past studies, evening type individuals has greater severity when compared to morning type individuals ${ }^{19}$. The current study went a step further to analyze the tinnitus severity in the morning and evening in both intermediate and moderate morning group.

Participants under intermediate group are those individuals who don't fall under morning or evening type. They have a very unstable circadian rhythm and that can be the reason why there was no difference in the severity in tinnitus in this group. So the result what we obtained can be considered as a floor effect. The difference in the tinnitus severity observed in the moderate morning individuals can be explained based on the secretion of melatonin hormone. Melatonin is a neuro hormone secreted by the pineal gland in the brain which controls the circadian rhythm in humans. The amount of melatonin secretion varies from one time point to another. Towards the evening, the secretion of melatonin is high and this increase in the quantity helps the individual to sleep. Towards the morning, the amount of melatonin reduces. Since the melatonin controls circadian rhythm, it can be assumed that secretion of such hormone induces sleepiness, drowsiness and reduced ability to attend to an activity and thereby enhancing the annoyance level of the person. This increase in the annoyance level may be the reason for the increased severity of tinnitus in the evening for the morning group individuals.

But when these individuals are active, especially in the morning, there is a reduction in the annoyance level and thereby experiences reduced tinnitus severity. Finding of the current study assumes that, if circadian rhythm has an influence on the tinnitus severity, altering the circadian type or chronotype of a tinnitus patient might change the severity of tinnitus. A future research has to focus on studying the tinnitus severity by changing the chronotypes of tinnitus sufferers. Also the current study didn't try to explain the difference in the tinnitus severity in other chronotypes such as definite morning, definite evening and moderate evening. This can be explained based on the fact that majority of the participants in the current study were in the age range of 55 years to 70 years. In this context, it is very unlikely to find an individual in this age range that will fall under definite or moderate evening group in Indian scenario. Future researches should consider individuals in this chronotypes also.

\section{CONCLUSION}

The results of the current study revealed that circadian rhythm has a strong association with the severity of the perceived tinnitus. The results of this preliminary study can be used in audiology clinics to explain to the tinnitus sufferers why they perceive difference in tinnitus severity across the day.

\section{Acknowledgement}

The authors would like to thank all the participants of the study for their participation, time and patience. The authors would also like to thank Shadiya, Jinsha, Prajakta, Antony, Mayur, Rekha Mam, Gayathri, and Archana Mam for their timely help and support.

\section{Conflict of Interest}

$\mathrm{NIL}$

\section{REFERENCES}

1. Han BI, Lee HW, Kim TY, Lim JS, Shin KS. Tinnitus: characteristics, causes, mechanisms, and treatments. J Clin Neurol. 2009;5(1):11-9. DOI:http://dx.doi.org/10.3988/jcn.2009.5.1.11

2. Jastreboff PJ, Hazell JW. A neurophysiological approach to tinnitus: clinical implications. Br J Audiol. 1993;27(1):7-17. PMID: 8339063

3. Axelsson A, Ringdahl A. Tinnitus--a study of its prevalence and characteristics. Br J Audiol. 1989;23(1):53-62. PMID: 2784987

4. Hoffman HJ, Reed GW. Epidemiology of tinnitus. In: Snow JB Jr, ed: Tinnitus: Theory and Management. Hamilton, Ontario: B.C. Decker, Inc.; 2004. p.16-41.

5. Tyler RS, Baker LJ. Difficulties experienced by tinnitus sufferers. J Speech Hear Disord. 1983;48(2):150-4. PMID: 6621006 DOI:http:// dx.doi.org/10.1044/jshd.4802.150

6. Udupi VA, Uppunda AK, Mohan KM, Alex J, Mahendra MH. The relationship of perceived severity of tinnitus with depression, anxiety, hearing status, age and gender in individuals with tinnitus. Int Tinnitus J. 2013;18(1):29-34. DOI: http://dx.doi.org/10.5935/09465448.20130005

7. Crocetti A, Forti S, Ambrosetti U, Bo LD. Questionnaires to evaluate anxiety and depressive levels in tinnitus patients. Otolaryngol Head Neck Surg. 2009;140(3):403-5. PMID: 19248952 DOI: http://dx.doi. org/10.1016/j.otohns.2008.11.036

8. Zhu L, Zee PC. Circadian rhythm sleep disorders. Neurol Clin. 2012;30(4):1167-91. DOI: http://dx.doi.org/10.1016/j. $\mathrm{ncl}$.2012.08.011

9. Stephan FK, Zucker I. Circadian rhythms in drinking behavior and locomotor activity of rats are eliminated by hypothalamic lesions. Proc Natl Acad Sci U S A. 1972;69(6):1583-6. PMID: 4556464 DOI: http://dx.doi.org/10.1073/pnas.69.6.1583

10. Green DJ, Gillette R. Circadian rhythm of firing rate recorded from single cells in the rat suprachiasmatic brain slice. Brain Res. 1982;245(1):198-200. PMID: 6889453 DOI: http://dx.doi. org/10.1016/0006-8993(82)90361-4

11. Hofman MA, Fliers E, Goudsmit E, Swaab DF. Morphometric analysis of the suprachiasmatic and paraventricular nuclei in the human brain: sex differences and age-dependent changes. J Anat. 1988;160:127-43. PMID: 3253251

12. Young ME, Bray MS. Potential role for peripheral circadian clock dyssynchrony in the pathogenesis of cardiovascular dysfunction. Sleep Med. 2007;8(6):656-67. DOI: http://dx.doi.org/10.1016/j. sleep.2006.12.010 
13. Klerman EB. Clinical aspects of human circadian rhythms. J Biol Rhythms. 2005;20(4):375-86. DOI: http://dx.doi. org/10.1177/0748730405278353

14. Horne JA, Ostberg O. A self-assessment questionnaire to determine morningness-eveningness in human circadian rhythms. Int J Chronobiol. 1976;4(2):97-110.

15. Di Milia L. Smith PA. Folkard S. A validation of the revised circadian type inventory in a working sample. Pers Individ Diff. 2005;39(7):1293305. DOI:http://dx.doi.org/10.1016/j.paid.2005.04.012
16. Smith CS, Reilly C, Midkiff K. Evaluation of three circadian rhythm questionnaires with suggestions for an improved measure of morningness. J Appl Psychol. 1989;74(5):728-38. PMID: 2793773 DOI: http://dx.doi.org/10.1037/0021-9010.74.5.728

17. Roberts RD, Irvine S. Construction and validation of the lark-owl (chronotype) indicator (LOCl): status report; 1999.

18. Roenneberg T, Wirz-Justice A, Merrow M. Life between clocks: daily temporal patterns of human chronotypes. J Biol Rhythms. 2003;18(1):80-90. DOI: http://dx.doi.org/10.1177/0748730402239679

19. Toplu Y, Selvi Y, Kizilay A. The role of individual biological rhythm differences and sleep quality on tinnitus symptom severity. Biol Rhythm Res. 2014;45(4):557-62. DOI: http://dx.doi.org/10.1080 /09291016.2013.873219 